\title{
Study of Possible Local Quasars I: The First Sample
}

\author{
K. P. Panov*
}

Institute of Astronomy, Bulgarian Academy of Sciences, 72, Tsarigradsko Shosse blvd, 1784 Sofia, Bulgaria

\begin{abstract}
This study provides new arguments for the existence of local quasars, i.e. quasars in the vicinity of low redshift galaxies. Local quasars are probably ejected from respective parent galaxy. The sample includes 74 quasars in the vicinity of 8 different galaxies. Assuming for quasars the same distances from the observer, as for their respective parent galaxy, simple calculations lead to the quasars luminosities and radii. Furthermore, the assumption is made that the major part of redshifts of quasars are due to gravitational reddening, i.e. they are intrinsic in origin. In this way, data for masses and densities of quasars are obtained and a diagram Density-Redshift is constructed. Relationships are also found for: Absolute mag. - Radius, Absolute mag - Mass, Mass - Radius, and Mass - Luminosity for this sample of local quasars. Comparison with the same diagrams for stars suggests a possible connection between stars and quasars. All these relationships are compelling evidence that the assumptions and the procedure in this study are correct. The relationships found imply that local quasars behave like single bodies, or at least the bulk of the quasar's mass is a single body, close to its gravitational radius. The theory of such strange bodies does not yet exist. Local quasars show signs of evolution: their redshifts decrease with time, as their densities decrease. The physics behind this evolution is not yet clear. However, yet unknown physical processes might be involved, which cause the ejection of quasars by active galactic nuclei and the subsequent disintegration of matter of quasars. The end-product of this evolution (disintegration) could be small-mass companion galaxies. A relation "mass - density" is found, which could be explained if the speed of evolution (disintegration) depends on the mass of the quasar: more massive quasars evolve more rapidly.
\end{abstract}

Keywords: Quasars, gravitational redshifts, quantization of redshifts, evolution of quasars.

\section{REMARKS ON HISTORY OF QUASARS}

Quasars (QSOs, quasi-stellar objects) have been discovered in 1963, following the optical identification of some radio-sources as quasi-stellar objects. Two objects, 3C273 and $3 \mathrm{C} 48$, revealed unusual and puzzling optical spectra, which were finally deciphered by M. Schmidt as being redshifted by $16 \%$ for $3 \mathrm{C} 273$ and by $37 \%$ for $3 \mathrm{C} 48$. Other discoveries of quasars followed and in all cases the large redshifts are present in their spectra. These large redshifts are unprecedented in astrophysics and remain a mystery to the present day. The total number of quasars now exceeds 130000. In the classical book of G. Burbidge and E.M. Burbidge, Quasi Stellar Objects (1967), following characteristics (specified already by M. Schmidt) are listed:

- $\quad$ star-like object, identified with a radio-source;

- variable light;

- $\quad$ large ultraviolet flux;

- $\quad$ large redshifts;

- broad emission lines in their spectra, with some absorption lines in some cases.

Later studies revealed that the majority of quasars do not radiate in the radio-band, some $90 \%$ of all quasars being

*Address correspondence to this author at the Institute of Astronomy, Bulgarian Academy of Sciences, 72, Tsarigradsko Shosse blvd, 1784 Sofia, Bulgaria; Tel/Fax: +359 2 9741910; E-mail: kpanov@astro.bas.bg radio-quiet. Typically, quasars show strong and variable $\mathrm{X}$ ray emission. Variability in several bands: optical, radio, and $\mathrm{X}$-ray, on short-time scales indicate that the emitting region in quasars should have small dimensions. For the large redshifts of QSOs, a number of possible causes have been put forward. It seems to me that four of them could be regarded as main candidates:

- Doppler shifts, due to relative motions. As QSOs exhibit always redshifts, we would have the unrealistic situation of quasars always receding from the observer. Thus this possibility is easily discarded, at least for the major part of the quasar-redshift. Some small Doppler-component in the QSO-redshift could, however, still be present.

- Gravitational reddening. It follows from the General Theory of Relativity.

Gravitational reddening is intrinsic in origin.

- "Intrinsic" redshift. There could be other than gravitational reddening causes for intrinsic redshifts. That is why some astronomers prefer not to specify the origin of the intrinsic redshift.

- Cosmological redshift. It follows from the expansion of the Universe.

During the past $\sim 40$ years, astrophysics witnessed a severe competition between the "cosmological" and the "intrinsic" interpretation of the redshifts The majority of astronomers seems to prefer the cosmological interpretation, which then puts quasars at great, cosmological distances and 
requires enormous energies to sustain their luminosities. In the next section, some of the problems, associated with the cosmological model for QSOs will be reviewed.

Presently, the most popular model of quasars is an accreting black hole, with a huge mass up to $10^{10} \mathrm{~m}_{\circ}$ (solar masses), at cosmological distance $[1,2]$. Recent general reviews are published by [3-5]. With the standard model, QSOs luminosities are huge: $\sim 10^{45} \mathrm{ergs} / \mathrm{s}$ in a life-time of $\sim$ $10^{7}-10^{8}$ years. The huge energy requirements of quasars eliminate early scenarios, such as "colliding galaxies", or "chains of supernovae explosions". We shall see in the following sections that the cosmological model for quasars (standard model) is not a matter of consensus. The first attempt to invoke a concept of a stellar-type objects for galactic nuclei with masses of $10^{5} \mathrm{~m}_{\circ}$ to $10^{8} \mathrm{~m}_{\circ}$ was done by Hoyle and Fowler in 1963 [6]. Here is a remark they made "...It is of course strange, but the very nature of the case demands an unusual physical situation". And another remark, made by Jeans in 1929: “...The type of conjecture which presents itself somewhat insistently is that the centers of nebulae (i.e. galaxies - my comment) are of the nature of singular points, at which matter is pouring into our universe..". Similar views have been put forward by Victor Ambartsumian about 60 years ago. He suggested that the unusual activity of galactic nuclei may be due to the disintegration of some dense matter of yet unknown origin and properties.

Are we closer now to these prophetic views?

\section{INTRODUCTION}

In this section, I shall review some of the problems, associated with the cosmological interpretation of QSOs redshifts.

- The huge luminosities of QSOs assuming cosmological distances present severe problems. The model requires a huge black hole and a huge amount of matter to be accreted, in order to sustain QSOs luminosities. The greater the distance to the quasar (distance increases with the redshift), the greater the luminosity of the quasar. This is a strange but unavoidable consequence of the standard model. The problem is, why we do not observe high luminosity QSOs at low redshifts? People try to explain it by rapid evolution of quasars. Strangely, the average luminosity of QSOs should decrease with time with just the necessary amount in order that their average apparent brightness at every cosmological distance remains about the same. What a queer coincidence. It still does not answer the question why there are no high luminosity QSOs at low redshifts.

- To avoid the high luminosity problem for distant quasars, it was suggested that there could be an enhancement due to gravitational lensing $[7,8]$. No evidence of such an enhancement was found. Unsuccessful turned also the attempts to reduce the high QSOs luminosities by assuming a "beam-radiation" (i.e. anisotropy of radiation). Statistical considerations clearly show that this scenario is impossible. The conclusion is, if QSOs are at cosmological distances, their luminosities should be huge and that is unavoidable.
- The standard model predicts that at earlier epochs (high z) QSOs should be more numerous and their number should increase with z. Observations show a peak of the QSOs-number at $\mathrm{z}=2.5-3$, and a decline at $\mathrm{z}=5$ and $\mathrm{z}=6$. Why is this decline? The standard model is unable to explain it.

- There is the so called "time-dilation effect" [9], which should be present in objects at cosmological distances, thus also in QSOs. Furthermore, "timedilation" should increase with z. No "time-dilation" in QSOs is observed, however [10].

- Studies showed that the redshifts of quasars often take preferred, specific values: 0.06, 0.30, 0.60, 0.96 and so on, which are obtained by the formula: $\Delta \log (1+\mathrm{z})$ $=0.089$. This is the so called Karlsson-sequence [1114]. Quantization of QSOs redshifts has no explanation with the standard model. Quantized cosmological redshifts would imply that the Universe expands "in shells" of different and specific velocities. Originally, the idea was conceived that the Karlsson's sequence may imply specific structure of the Universe, where shells of increased densities occur at specific distances (and velocities!) from the observer. It was soon realized that such a structure contradicts the cosmological principle - it is quite unrealistic. Presently, there is some doubt on the reality of the Karlsson's sequence. It should be realized, however, that quasarredshifts are probably "a mixture" of components of different origins. This could explain the negative result in some recent studies. We shall see below that the problem of quantized redshifts could be handled if the redshifts are intrinsic.

- There is an old problem for the standard model - the association of high redshift QSOs with low redshift galaxies [15-19]. An excellent, compelling evidence for a great number of such discordant redshift associations is the "Catalogue of discordant redshift associations" by Halton Arp [16]. Prominent examples are the NGC4319 and Mk205 with a luminous filament between them, the association of NGC3067 and 3C232, the NGC7603 and NGC7603B etc. In some "discordant redshift associations" there are connecting filaments (optical or radio), showing the vicinity in space of their members. In other cases, quasars have been found just a couple of arcsec from the respective galaxy. Consideration of chance-projection in these cases show a probability of less than $10^{-8}$ [20]. In some associations, quasars or X-ray-sources are found as pairs "across" a Seyfert galaxy [21]. In other cases, there are a number of quasars aligned in the vicinity of a Seyfert galaxy, and the alignment often coincides with the minor (rotational) axis of the nearby galaxy [22,23]. In all cases, showing alignment of quasars, the probability of a chance projection decreases dramatically, practically an impossible situation [24]. Clearly, if quasars and galaxies of different redshifts are associated in space, the redshifts of quasars could not be cosmological. A single case of such a "discordant redshift association" is enough to be a proof. An amazing development in recent years is the discovery of groups of quasars in the vi- 
cinity of active, usually Seyfert-type low redshift galaxies (see Table 1 below). Even if there is no visible physical connection between a galaxy and nearby quasars, the existence of a group of QSOs close to a galaxy on the sky makes the case of projection by chance extremely unlikely, practically impossible. The problem has been discussed many times in the past and I am not going to argue about it here. Indeed, a group of quasars physically associated with a galaxy requires explanation. Such an association could not be built by a gravitational collapse, I believe. The alternative is that QSOs have been ejected by the active galactic nuclei [25-28]. The physics of this ejection is not yet clear and could lead to exciting new developments in astrophysics and cosmology.

- A final problem that I would like to point out is the apparent high metallic content in quasars. Since QSOs are believed by the standard model to be at cosmological distances, they should be young objects. The larger the redshift, the younger the quasar. Therefore, quasars should be deficient in metals and a gradient should be observed of the metallic abundances with z. Observations show no metal deficiency in quasars and no gradient of metallicity [29,30]. Quite a surprising result!

The gravitational reddening as a possible cause for QSOs redshifts has been considered very early in the quasarhistory, but then abandoned for various reasons [31]. Another popular hypothesis for the intrinsic origin of the redshifts is the "variable mass hypothesis". [32,33].

In the following sections, I will try to revive the gravitational reddening as primary origin for the QSOs redshifts.

\section{THE SAMPLE OF QUASARS}

During the last years, a number of studies have been published on possible associations of quasars and low redshift galaxies (usually, Seyfert-type). In some cases, groups of up to 20 and more QSOs have been reported close to a galaxy. In all these studies, the authors provide convincing arguments that such associations could not be observed by a chance projection. The sample of local quasars used in this study is listed in Table $\mathbf{1}$. Angular distances of these quasars from each respective galaxy are not greater than 2 degrees.

Altogether, 74 quasars have been chosen around 8 different galaxies. Table 1 lists their redshifts, visual magnitudes and colours, taken from Veron-Cetty and Veron [34]. If some quasar was included in this sample by chance projection (although this is very unlikely), I would expect that in the following analysis this quasar will show up by some deviation from the general behaviour of the rest of the QSOssample. Quasars in the region of NGC2639 were suspected to have been ejected by a companion galaxy near NGC2639, and not by NGC2639 itself [40]. This claim has not been corroborated by later studies. A companion galaxy would be at about the same distance as NGC2639, and quasar's characteristics would not change very much if this is the case. If, however, the parent galaxy is not NGC2639 and not a companion of NGC2639, but these quasars are at substantially different distances, the group of quasars for NGC2639 (Table 1) would have different characteristics.

\section{THE PROCEDURE AND THE RESULTS.}

In this study, I will proceed in the following way. Several assumptions will be made in order to obtain the physical characteristics of the sample-quasars: luminosities, radii, masses, and densities. Relationships between physical characteristics will be established on the basis of the assumptions made. The existence of well defined relationships will be considered as confirmation of these assumptions.

Quasar luminosities are easily obtained, taking as distance to the respective quasar the distance to its parent galaxy. This follows from the assumption that groups of quasars (Table 1) and respective close-by galaxy are spatially associated.

Quasars radii are calculated from:

$\log \left(\mathrm{r}_{\mathrm{q}} / \mathrm{r}_{\circ}\right)=1 / 2 \log \left(\mathrm{L}_{\mathrm{q}} / \mathrm{L}_{\circ}\right)+2 \log \left(\mathrm{T}_{\circ} / \mathrm{T}_{\mathrm{q}}\right)$

where $\mathbf{r}, \mathbf{L}$, and $\mathbf{T}$ are radii, luminosities, and temperatures, respectively. Symbols "q" and "o" stay for quasars and the Sun, respectively. The above formula implies that the bulk of quasar-mass is contained in a single body. Temperatures of quasars in eq. (1) are determined by their B-V colours.

The redshifts of quasars are taken as composed by three components, according to Burbidge [41]:

$\left(1+z_{0}\right)=\left(1+z_{c}\right) \cdot\left(1+z_{i}\right) \cdot\left(1+z_{d}\right)$

where: $z_{0}$ - is observed redshift

$\mathrm{z}_{\mathrm{c}}$ - cosmological redshift

$\mathrm{z}_{\mathrm{i}}$ - intrinsic redshift

$z_{d}$ - Doppler redshift

The disentanglement of each component from the observed value is not an easy task. The exact disentanglement is not possible at present and in the following I will try only a "first step approach", being aware that possible errors may be introduced. The cosmological redshift of quasars is taken here to be the redshift of the corresponding parent galaxy. In order to reduce redshifts to the respective galaxy (thus make a reduction for cosmological redshift), all quasar-redshifts are re-calculated as follows:

$\mathrm{z}_{\mathrm{i}}=\left(\mathrm{z}_{\mathrm{o}}-\mathrm{z}_{\mathrm{gal}}\right) /\left(1+\mathrm{z}_{\mathrm{gal}}\right)$

with $\mathrm{z}_{\mathrm{gal}}=\mathrm{z}_{\mathrm{c}}$ being the redshift of the respective galaxy. With this definition, $z_{i}$ contains also the Doppler redshiftcomponent, which is presently unknown. In the next step, each intrinsic redshift is compared with the Karlsson'ssequence: $0.06,0.30,0.60,0.96,1.41,1.96, \ldots$ etc, and the nearest value of this sequence is taken to replace the $z_{i}$. In this way, the assumption is taken that intrinsic redshifts are "quantized". In the previous section, I discussed the problem of quantized cosmological redshifts. There are no a-priori arguments against the possibility of quantized intrinsic redshifts. The discrepancy between the actual $\mathrm{z}_{\mathrm{i}}$ and the respective nearest value from the Karlsson's sequence could be attributed to the presence of the Doppler component (e.g. velocity of ejection). Doppler redshift components $z_{d}$ will not be discussed further in this study. At this point, I am going to make an important assumption: the intrinsic redshifts are due to gravitational reddening, i.e. $\mathrm{z}_{\mathrm{i}}=\mathrm{z}_{\mathrm{gr}}$

For gravitational reddening, 
Table 1. Sample of Local Quasars, Data from the Catalogue of Veron-Cetty and Veron, 13 th.ed [34]

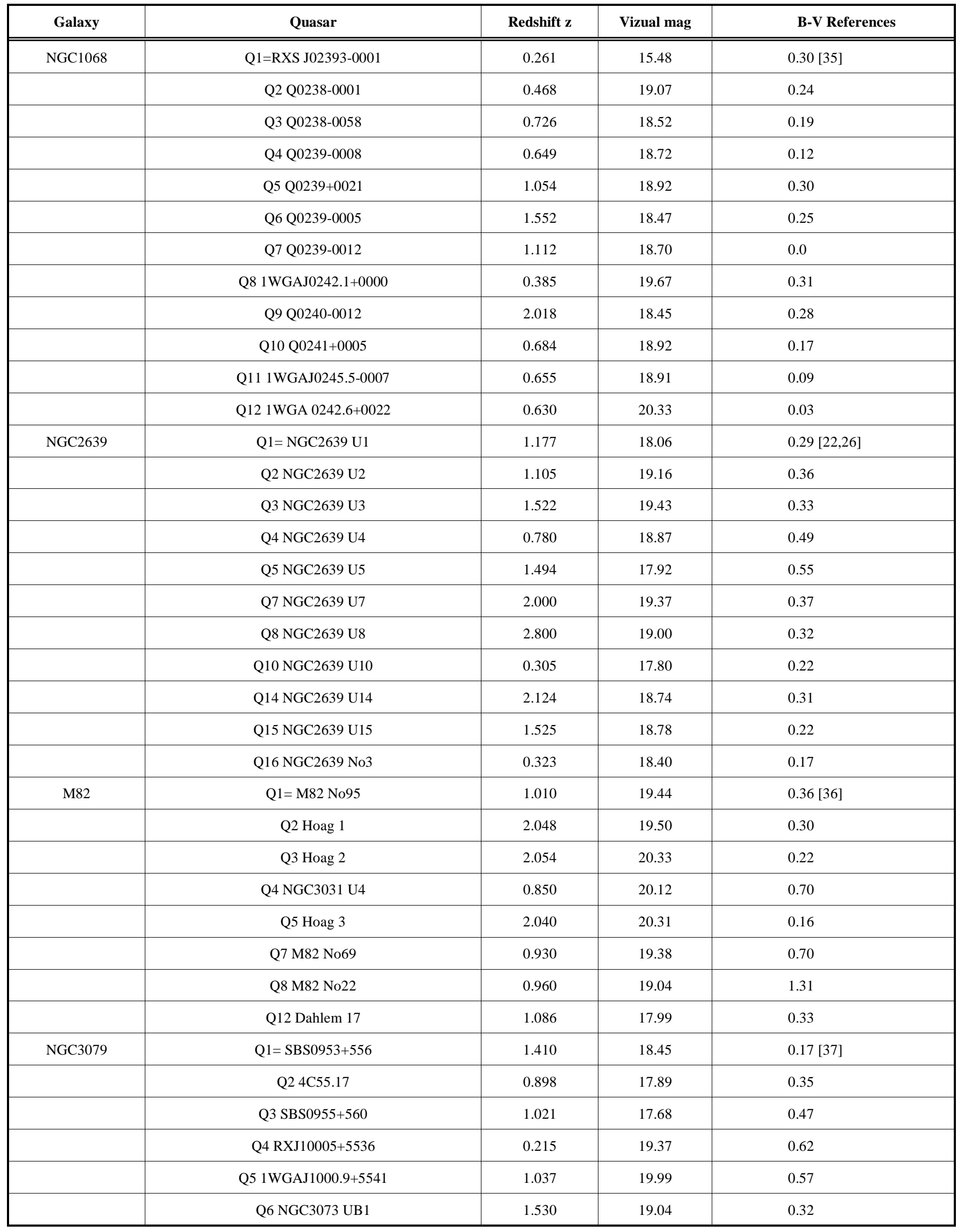


Table 1. contd....

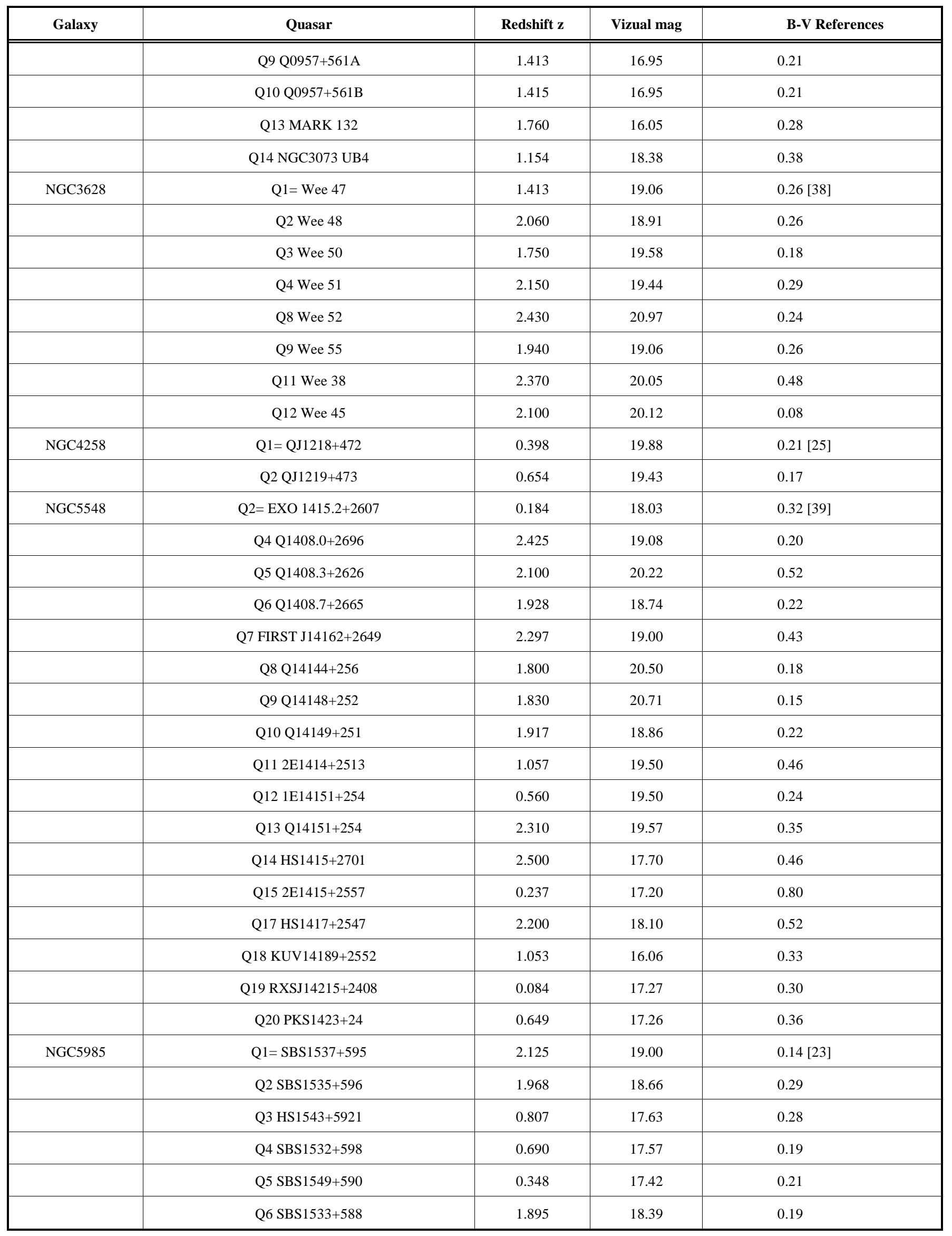




$$
\left(1+\mathrm{z}_{\mathrm{gr}}\right)=\left(1-\mathrm{r}_{\mathrm{gr}} / \mathrm{r}_{\mathrm{q}}\right)^{-1 / 2}
$$

Substituting in eq. (4) the value of $\mathrm{z}_{\mathrm{gr}}=\mathrm{z}_{\mathrm{i}}$, we can obtain the ratio $r_{g r} / r_{q}$, and with $r_{q}$ already calculated we get the gravitational radii $r_{g r}$ of quasars. For the gravitational radius holds:

$$
\mathrm{r}_{\mathrm{gr}}=\left(2 \mathrm{Gm}_{\mathrm{q}}\right) / \mathrm{c}^{2}
$$

where $\mathbf{G}$ is gravitational constant, $\mathbf{m}_{\mathbf{q}}$ is the mass of the quasar, and $\mathbf{c}$ is the velocity of light. Thus the approach taken makes it possible with simple calculations to obtain the quasar-mass $\mathbf{m}_{\mathbf{q}}$ and the quasar-density $\boldsymbol{\rho}_{\mathbf{q}}$. In Table $\mathbf{2}$, columns are self-explanatory. The column $\boldsymbol{\rho}^{\sim}$ will be explained later.

Table 2. Physical Characteristics of Local Quasars

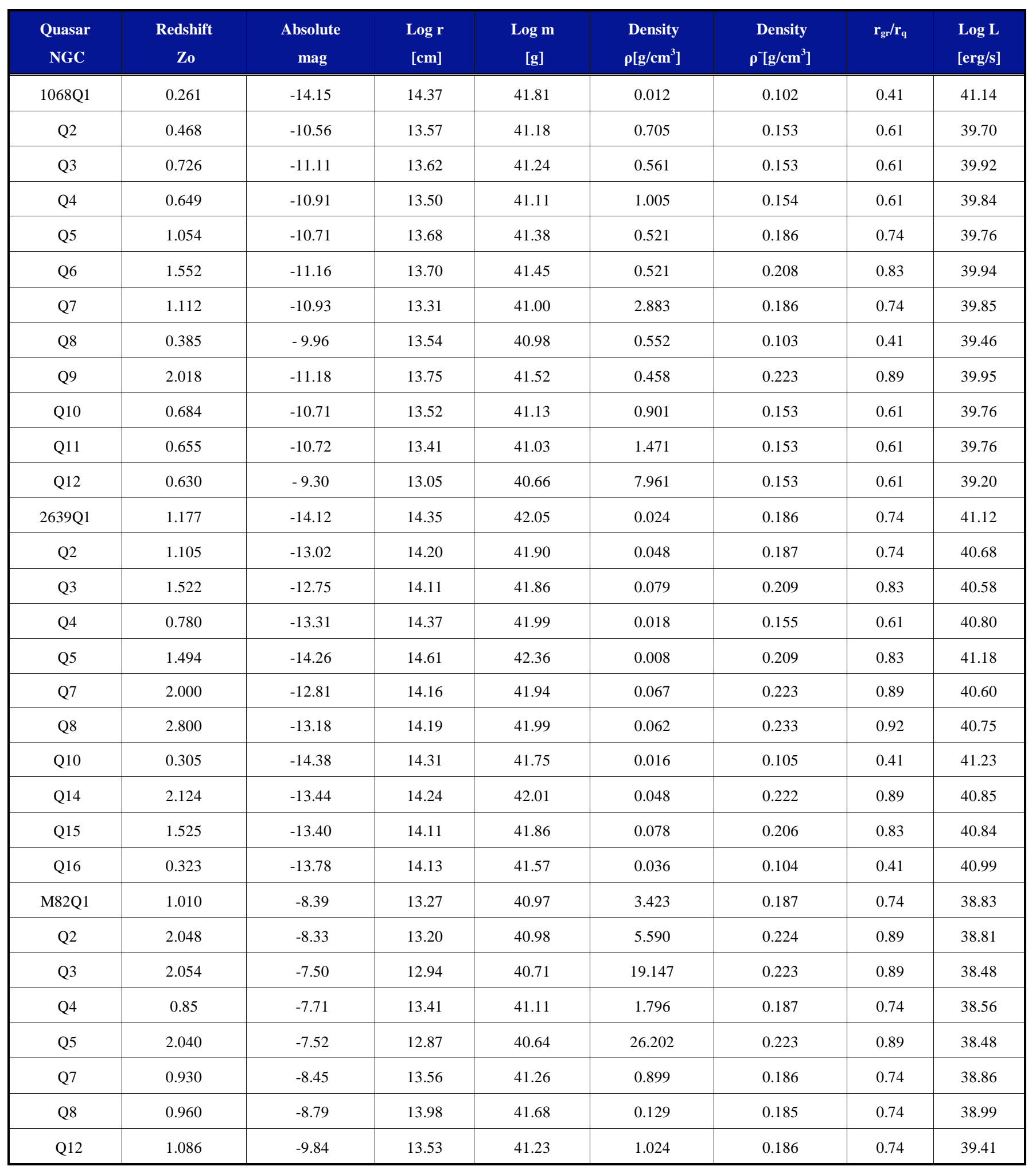


Table 2. contd....

\begin{tabular}{|c|c|c|c|c|c|c|c|c|}
\hline $\begin{array}{c}\text { Quasar } \\
\text { NGC }\end{array}$ & $\begin{array}{c}\text { Redshift } \\
\text { Zo }\end{array}$ & $\begin{array}{c}\text { Absolute } \\
\text { mag }\end{array}$ & $\begin{array}{r}\log \mathbf{r} \\
{[\mathrm{cm}]}\end{array}$ & $\begin{array}{c}\log \mathbf{m} \\
{[\mathrm{g}]}\end{array}$ & $\begin{array}{c}\text { Density } \\
\rho\left[\mathrm{g} / \mathrm{cm}^{3}\right]\end{array}$ & $\begin{array}{c}\text { Density } \\
\rho\left[\mathrm{g} / \mathrm{cm}^{3}\right]\end{array}$ & $\mathbf{r}_{\mathrm{gr}} / \mathbf{r}_{\mathrm{q}}$ & $\begin{array}{c}\text { Log L } \\
{[\mathrm{erg} / \mathrm{s}]}\end{array}$ \\
\hline 3079Q1 & 1.410 & -11.83 & 13.74 & 41.49 & 0.435 & 0.208 & 0.83 & 40.21 \\
\hline Q2 & 0.898 & -12.39 & 14.06 & 41.76 & 0.090 & 0.186 & 0.74 & 40.43 \\
\hline Q3 & 1.021 & -12.60 & 14.21 & 41.91 & 0.045 & 0.187 & 0.74 & 40.52 \\
\hline Q5 & 1.037 & -10.29 & 13.84 & 41.53 & 0.253 & 0.186 & 0.74 & 39.59 \\
\hline Q6 & 1.530 & -11.24 & 13.80 & 41.55 & 0.329 & 0.208 & 0.83 & 39.97 \\
\hline Q9 & 1.413 & -13.33 & 14.09 & 41.84 & 0.088 & 0.208 & 0.83 & 40.81 \\
\hline Q10 & 1.415 & -13.33 & 14.09 & 41.84 & 0.088 & 0.208 & 0.83 & 40.81 \\
\hline 3628Q1 & 1.413 & -11.24 & 13.73 & 41.48 & 0.456 & 0.209 & 0.83 & 39.97 \\
\hline Q2 & 2.060 & -11.39 & 13.76 & 41.54 & 0.425 & 0.223 & 0.89 & 40.03 \\
\hline Q3 & 1.750 & -10.72 & 13.53 & 41.31 & 1.231 & 0.222 & 0.89 & 39.76 \\
\hline Q4 & 2.150 & -10.86 & 13.70 & 41.47 & 0.579 & 0.223 & 0.89 & 39.82 \\
\hline Q8 & 2.430 & -9.33 & 13.33 & 41.12 & 3.325 & 0.233 & 0.92 & 39.21 \\
\hline Q9 & 1.940 & -11.24 & 13.73 & 41.51 & 0.488 & 0.223 & 0.89 & 39.97 \\
\hline Q11 & 2.370 & -10.25 & 13.75 & 41.55 & 0.468 & 0.233 & 0.92 & 39.58 \\
\hline Q12 & 2.100 & -10.18 & 13.29 & 41.06 & 3.755 & 0.223 & 0.89 & 39.55 \\
\hline Q5 & 2.100 & -13.41 & 14.42 & 42.19 & 0.021 & 0.224 & 0.89 & 40.84 \\
\hline Q6 & 1.928 & -14.89 & 14.41 & 42.19 & 0.021 & 0.220 & 0.89 & 41.43 \\
\hline Q7 & 2.297 & -14.63 & 14.59 & 42.36 & 0.010 & 0.233 & 0.89 & 41.33 \\
\hline Q8 & 1.800 & -13.13 & 14.01 & 41.79 & 0.134 & 0.222 & 0.89 & 40.73 \\
\hline Q9 & 1.830 & -12.92 & 13.94 & 41.71 & 0.189 & 0.222 & 0.89 & 40.64 \\
\hline Q10 & 1.917 & -14.77 & 14.39 & 42.16 & 0.024 & 0.225 & 0.89 & 41.38 \\
\hline Q11 & 1.057 & -14.13 & 14.51 & 42.21 & 0.011 & 0.180 & 0.74 & 41.13 \\
\hline Q12 & 0.560 & -14.13 & 14.29 & 41.90 & 0.026 & 0.151 & 0.61 & 41.13 \\
\hline Q13 & 2.310 & -14.06 & 14.40 & 42.17 & 0.023 & 0.223 & 0.89 & 41.10 \\
\hline Q14 & 2.500 & -15.93 & 14.87 & 42.67 & 0.003 & 0.259 & 0.92 & 41.85 \\
\hline Q15 & 0.237 & -16.43 & 15.23 & 42.67 & 0.0002 & 0.090 & 0.41 & 42.05 \\
\hline Q17 & 2.200 & -15.53 & 14.84 & 42.62 & 0.003 & 0.225 & 0.89 & 41.69 \\
\hline Q18 & 1.053 & -17.57 & 15.08 & 42.78 & 0.0008 & 0.180 & 0.74 & 42.50 \\
\hline Q19 & 0.084 & -16.36 & 14.81 & 41.68 & 0.0004 & 0.026 & 0.11 & 42.02 \\
\hline Q20 & 0.649 & -16.37 & 14.87 & 42.48 & 0.002 & 0.170 & 0.61 & 42.02 \\
\hline
\end{tabular}


Table 2. contd....

\begin{tabular}{|c|c|c|c|c|c|c|c|c|}
\hline $\begin{array}{c}\text { Quasar } \\
\text { NGC }\end{array}$ & $\begin{array}{c}\text { Redshift } \\
\text { Zo }\end{array}$ & $\begin{array}{c}\text { Absolute } \\
\text { mag }\end{array}$ & $\begin{array}{r}\log r \\
{[\mathrm{~cm}]}\end{array}$ & $\begin{array}{c}\log \mathbf{m} \\
{[\mathrm{g}]}\end{array}$ & $\begin{array}{c}\text { Density } \\
\rho\left[\mathrm{g} / \mathrm{cm}^{3}\right]\end{array}$ & $\begin{array}{c}\text { Density } \\
\rho^{\sim}\left[\mathrm{g} / \mathrm{cm}^{3}\right]\end{array}$ & $\mathbf{r}_{\mathrm{gr}} / \mathbf{r}_{\mathrm{q}}$ & $\begin{array}{c}\log \mathrm{L} \\
\text { [erg/s] }\end{array}$ \\
\hline 5985Q1 & 2.125 & -12.62 & 13.87 & 41.64 & 0.263 & 0.223 & 0.89 & 40.52 \\
\hline Q2 & 1.968 & -12.96 & 14.12 & 41.89 & 0.084 & 0.225 & 0.89 & 40.66 \\
\hline Q3 & 0.807 & -13.99 & 14.31 & 42.01 & 0.029 & 0.189 & 0.74 & 41.07 \\
\hline Q4 & 0.690 & -14.05 & 14.21 & 41.82 & 0.037 & 0.152 & 0.61 & 41.10 \\
\hline Q5 & 0.348 & -14.20 & 14.26 & 41.70 & 0.020 & 0.105 & 0.41 & 41.16 \\
\hline Q6 & 1.895 & -13.23 & 14.05 & 41.82 & 0.116 & 0.223 & 0.89 & 40.77 \\
\hline
\end{tabular}

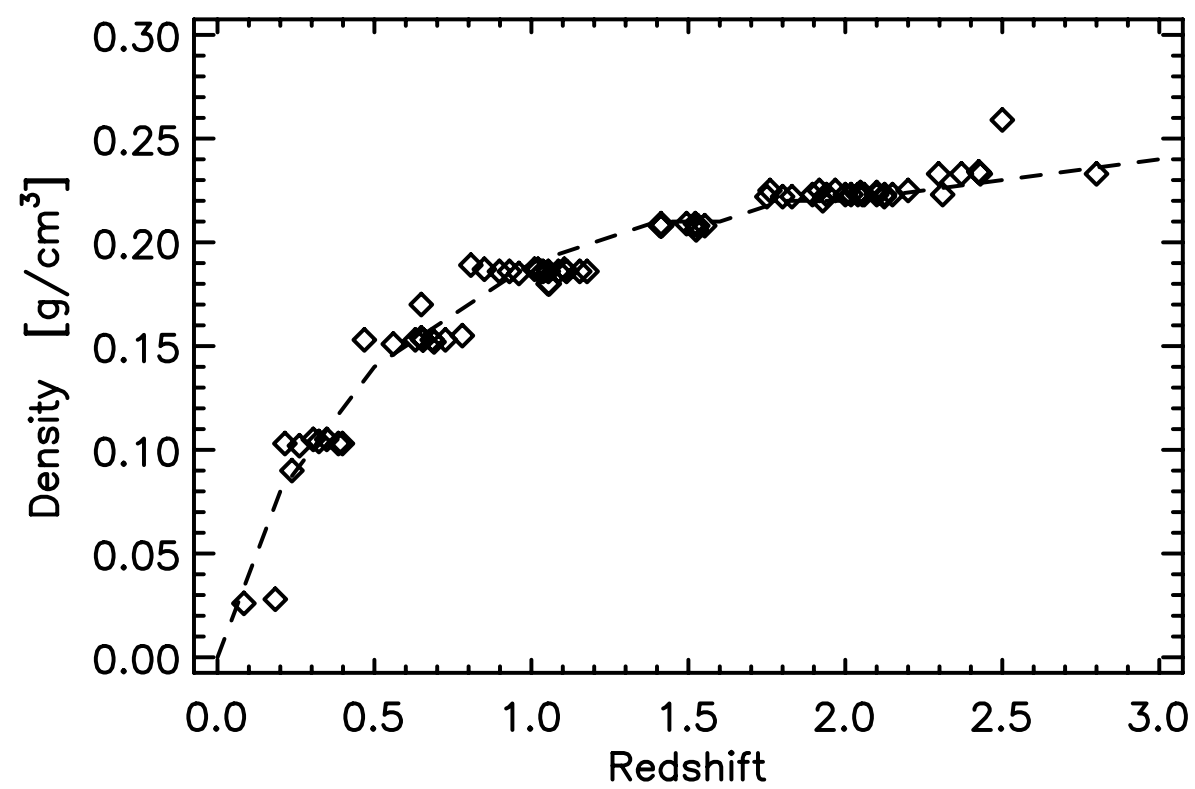

Fig. (1). "Density- observed redshift" diagram for the sample of quasars. All densities are reduced to a radius of $8 \times 10^{13} \mathrm{~cm}$. The dashed line is the theoretical line from eq. (6) for radius $8 \times 10^{13} \mathrm{~cm}$.

From Table 2, following ranges for the sample-quasars are apparent: absolute mags in -8 to -18 , radii in $10^{13} \mathrm{~cm}$ to $2 \times 10^{15} \mathrm{~cm}$, masses in $20.10^{6} \mathrm{~m}_{\circ}-3000.10^{6} \mathrm{~m}_{\circ}$, luminosities in $3.10^{38} \mathrm{erg} / \mathrm{s}$ to $3.10^{42} \mathrm{erg} / \mathrm{s}$, and densities in 0.0002 to 26 $\mathrm{g} / \mathrm{cm}^{3}$. Before we go to the graphics, one more relation should be shown. If we combine equations (4) and (5) with the obvious formula: $\mathrm{m}_{\mathrm{q}}=4 / 3 \pi \mathrm{r}_{\mathrm{q}}{ }^{3} \rho_{\mathrm{q}}$, we get for the density $\boldsymbol{\rho}_{\mathbf{q}}$ the expression:

$$
\rho_{\mathrm{q}}=(3 /(4 \pi)) \cdot\left(\mathrm{c}^{2} /(2 \mathrm{G})\right) \cdot\left(1 / \mathrm{r}_{\mathrm{q}}{ }^{2}\right) \cdot\left\{1-\left[1 /\left(1+\mathrm{z}_{\mathrm{gr}}\right)^{2}\right]\right\}
$$

From eq. (6), the density $\boldsymbol{\rho}_{\mathbf{q}}$ depends not only on the intrinsic redshift $\mathbf{z}_{\mathbf{g r}}$, but also on the inverse square of $\mathbf{r}_{\mathbf{q}}$. Thus if we try to plot the data for densities (Table 2) versus redshifts, there would be a great spread of data because of the dependence on radius. We could avoid this obstacle and obtain a more instructive diagram in the following way. Since we assumed that all sample-quasars behave according to eq.(6), we could reduce all densities to some radius of choice, say $r_{q}=8.10^{13} \mathrm{~cm}$. The reader may choose a different radius for reduction, it should not change the conclusions. Each density should be reduced with its own factor, determined by the individual radius of the quasar. The reduced densities for QSOs are given in Table 2. in the $\boldsymbol{\rho}^{\sim}$ column and plotted against the observed redshifts in Fig. (1). Data for redshifts are used from Table 2. Fig. (1) is a strong piece of evidence and needs an explanation. The fit of the sample of 74 quasars to the theoretical line for $8.10^{13} \mathrm{~cm}$ is obvious. This fit could not be due to a coincidence. Therefore, Fig. (1) is consistent with all assumptions and the procedure applied. It is in fact a confirmation of the procedure.

From Fig. (1), apparently density goes asymptotically to a limit with increasing $\mathrm{z}_{\mathrm{O}}$, in this case the limit is $\sim 0.252$ $\mathrm{g} / \mathrm{cm}^{3}$. The general expression for this limit is obtained from eq.(6), if $z_{\mathrm{gr}}=\infty$ :

$$
\left.\rho_{\text {limit }}=(3 /(4 \pi)) \cdot\left(c^{2} / 2 G\right)\right) \cdot\left(1 / r_{\mathrm{q}}{ }^{2}\right)
$$

What could be the physical implication of Fig. (1). I believe, this diagram shows possible picture of evolution: the redshifts will decrease as quasar densities decrease. First of all, is there an observational evidence of redshifts evolving with time? In a number of papers [28,42,43, and references therein], Halton Arp suggested an evolutionary scenario for quasars, where redshifts decrease as quasars recede from a parent galaxy. This scenario is based on the hypothesis of subsequent ejection of several quasars from the same parent galaxy. A nice example may be NGC5985, where a sequence of 5 QSOs is found with increasing distances from this galaxy. Distances of quasars from the parent galaxy, inferred by 


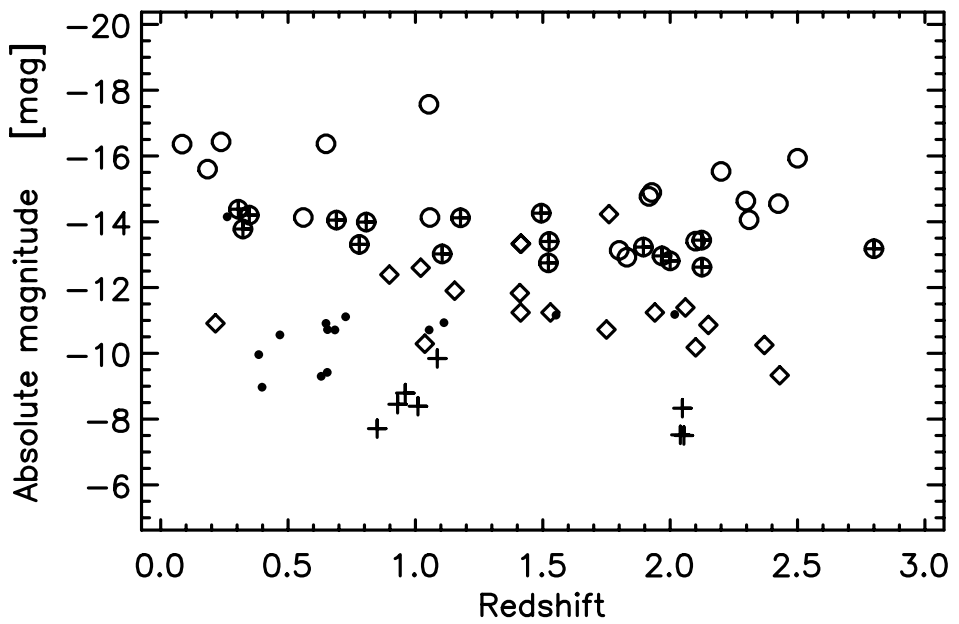

Fig. (2). Quasar absolute magnitude versus observed redshift. Symbols used are: crosses - M82; dots - NGC1068 and NGC4258; rhombs NGC3628 and NGC3079; encircled crosses - NGC2639 and NGC5985; circles - NGC5548. Note the separation in absolute magnitude for quasars of different galaxies.

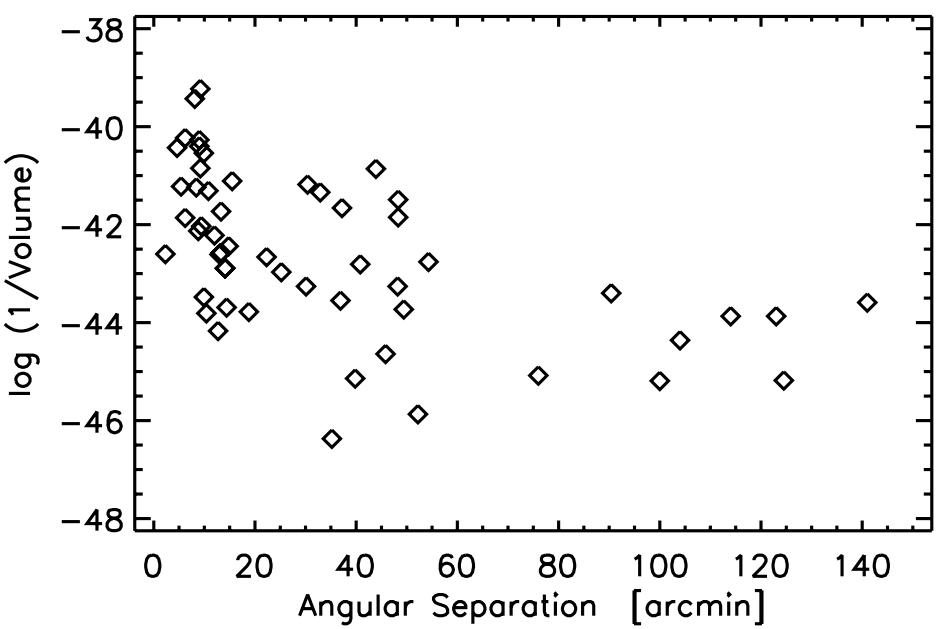

Fig. (3). Relationship between quasar-volume $\left[i n \mathrm{~cm}^{3}\right]$ and angular separation (in arcmin) from respective galaxy. Quasars of NGC2639 and NGC3628 are not included because of insufficient data.

their angular separation, increase with time (ejection hypothesis). Then the evolution of parameters of these quasars with respect to angular separation should be considered as a time-dependence. From Table 2, apparently, the densities of the NGC5985-quasars also show a trend: densities decrease with time. This finding is consistent with Fig. (1) and could be regarded as a hint of support of Arp's evolutionary scenario. The example of NGC5985 is, of course, insufficient to prove this scenario.

The end-result of the evolution of quasars in the Arp's scenario are galaxies. Looking at the masses in Table 2, I would say, they should be low mass galaxies, or companion galaxies. They have low densities, low redshifts, but higher luminosities. Thus increasing luminosities in Arp's scenario is predicted and one could try to observe it. In Fig 2, absolute mags of the sample quasars are plotted against the observed redshift.

Different signs are used for the quasars of different galaxies. Clearly, quasars from different galaxies are separated in luminosities, the M82 quasars being less luminous, and the NGC5548 being most luminous in our sample. This should be due to the difference of their radii (Table 2 ), the NGC5548 quasars being systematically larger. It is not clear why this separation in radii and luminosities occur, but this feature makes the search for an evolutionary trend of the quasar luminosity more difficult. Indeed, some of the sequences in Fig. (2) may show a trend of increasing luminosity, due to the evolution of redshift to lower values.

As an additional argument for evolution with decreasing density, characteristics of quasars could be studied with respect to their angular separation from the respective galaxy. This may bring new difficulties, however. In addition to the different radii, conditions in the surroundings of different galaxies are likely to be different: acceleration of gravity, the speed of ejection, etc. All these factors are expected to contribute to a larger scatter, if we combine the quasars from different galaxies.

In Fig. (3), the inverse volume of the sample-quasars is plotted against the angular separation for each quasar of Table 1, except for the group of NGC2639 and NGC3628. Despite of the large scatter, some trend of the quasar volume with separation (i.e. with time), could possibly exist, in the sense that volume possibly increases and therefore density decreases with time (masses of quasars presumed not changing). This result would be consistent with the Arp,s evolutionary scenario, because decreasing densities with time 


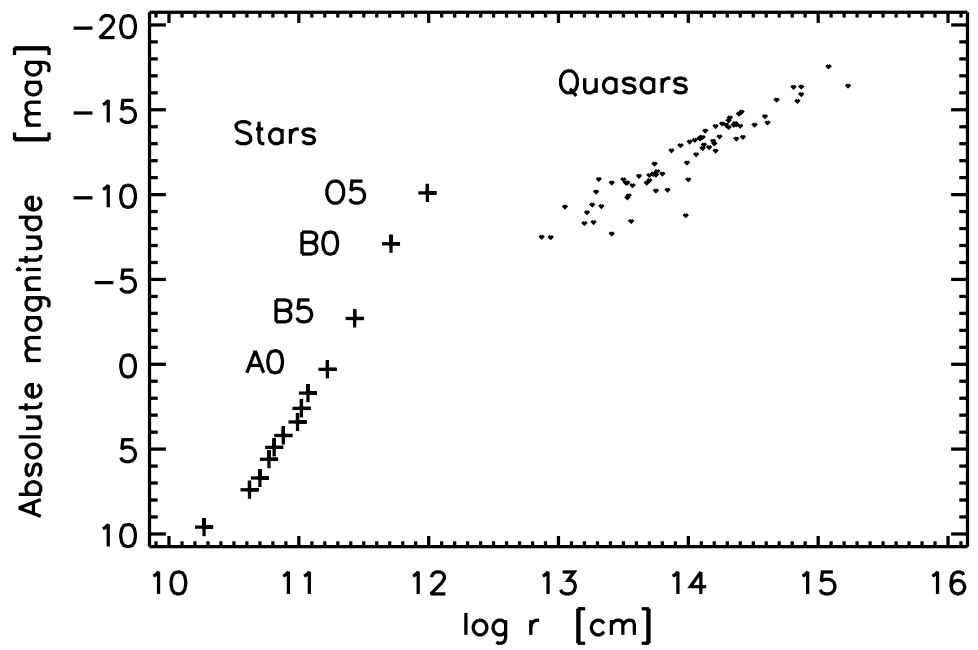

Fig. (4). Diagram "absolute magnitude - radius" for the sample of quasars (dots). The same relationship is shown also for stars (crosses), as mean values for O5, B0, B5,....,M5.

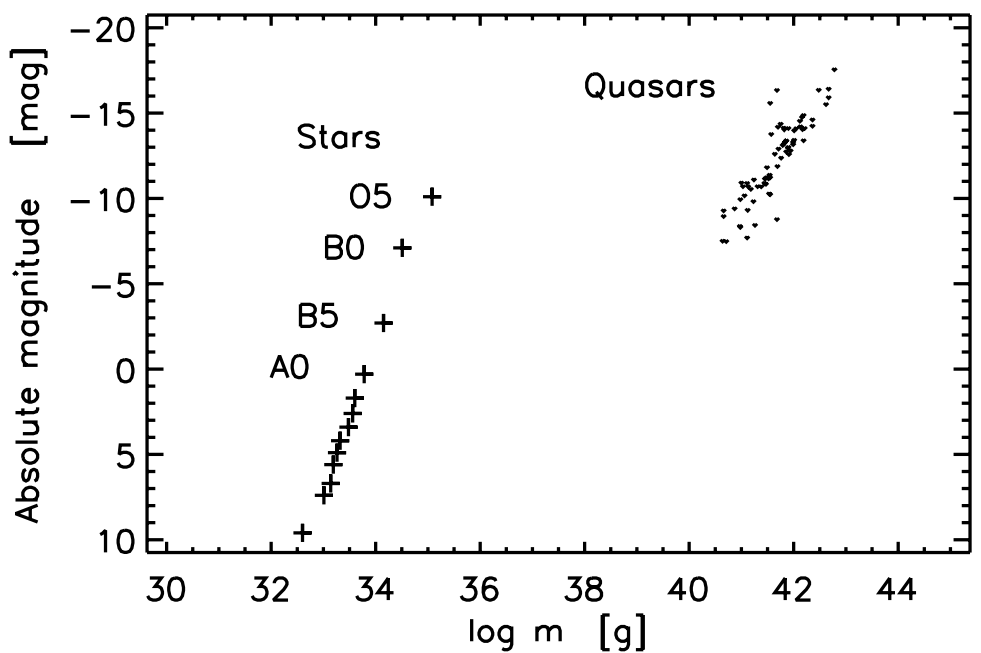

Fig. (5). Diagram "absolute magnitude - mass" for the sample of quasars (dots). The same relationship is also shown for stars (crosses), as mean values for: O5, B0, B5,....,M5.

means also decreasing redshifts. However, in view of its importance, further studies are necessary.

Fig. (1) shows one more interesting feature. For large redshifts, small variations of the density cause large variations in the redshifts. Thus if we assume the evolution with decreasing density, evolution presumably starts with high density and redshift. Then only a small drop in density would cause a large decrease in redshift. Is this the reason why very large redshifts are not observed? In the Introduction, the problem was mentioned concerning the decline of number of quasars with $z>3$. Fig. (1) could provide the answer: at large redshifts, the evolution to lower values of redshift as density decreases is very fast.

I could not avoid here the important question, why should densities of quasars decrease with time? Is this, perhaps, the "super-dense matter", suggested by Victor Ambartsumian? Do we observe a spontaneous disintegration of matter of yet unknown origin and properties? It may be so. That is why studies of quasars are so important.

Fig. (4) shows the diagram "absolute mag - radius" for the sample of quasars (data from Table 2). The same relationship is shown also for the stars O5 -M5. Linear ap- proximation (in coordinates: Absolute mag - log r) seems possible for this sample of quasars (least squares solution):

$\mathrm{M}_{\mathrm{q}}=48.099-4.318 . \log \mathrm{r}_{\mathrm{q}}$, with correlation coeff: -0.94

In Fig. (5), absolute mags are plotted against the masses (data from Table 2). Apparently, an "Absolute mag - mass" relation exists also for quasars. The linear approximation (in coordinates: Absolute mag - $\log \mathrm{m}$ ) is:

$\mathrm{M}_{\mathrm{q}}=158.808-4.107 . \log \mathrm{m}_{\mathrm{q}}$, with correlation coeff: -0.88

In Fig. (6), the "mass-radius " relation is shown (data from Table 2). The linear approximation is:

$\log \mathrm{m}_{\mathrm{q}}=28.692+0.927 . \log \mathrm{r}_{\mathrm{q}}$, with correlation coeff: $0.94(10)$

In 1964, J.L. Greenstein and M. Schmidt, discussing the possibility of gravitational redshifts in quasars wrote: “...If stable, massive configurations exist, we must re-examine this possibility. The mass-radius relation would have to be such as to give larger gravitational redshifts for fainter objects" [31].

Indeed, with eq. (10) this condition is fulfilled: fainter quasars have larger gravitational redshifts. A word of caution: corroboration by a larger sample of local quasars is 


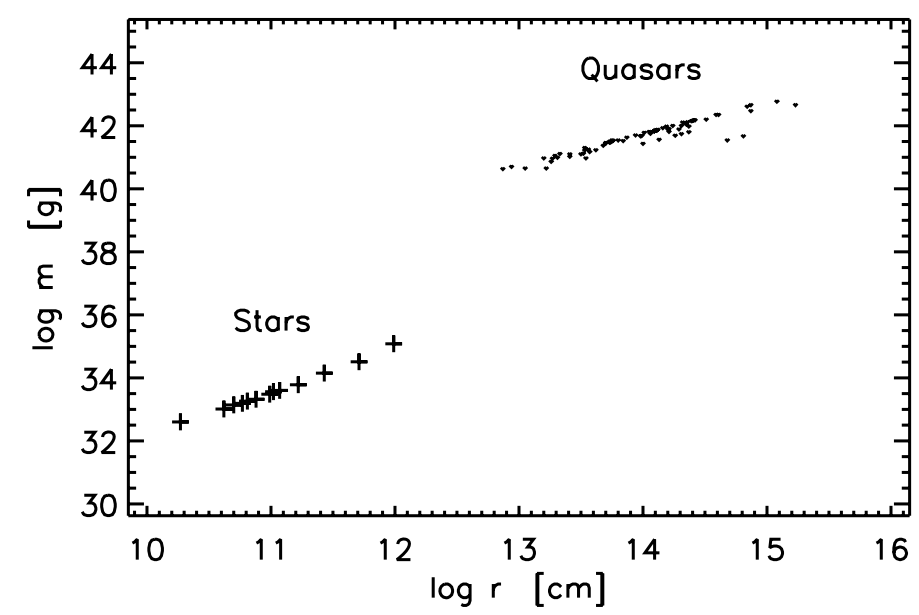

Fig. (6). Diagram "mass - radius" for the sample of quasars (dots). The same relationship is shown also for stars (crosses), as mean values for: O5, B0, B5,...,M5.

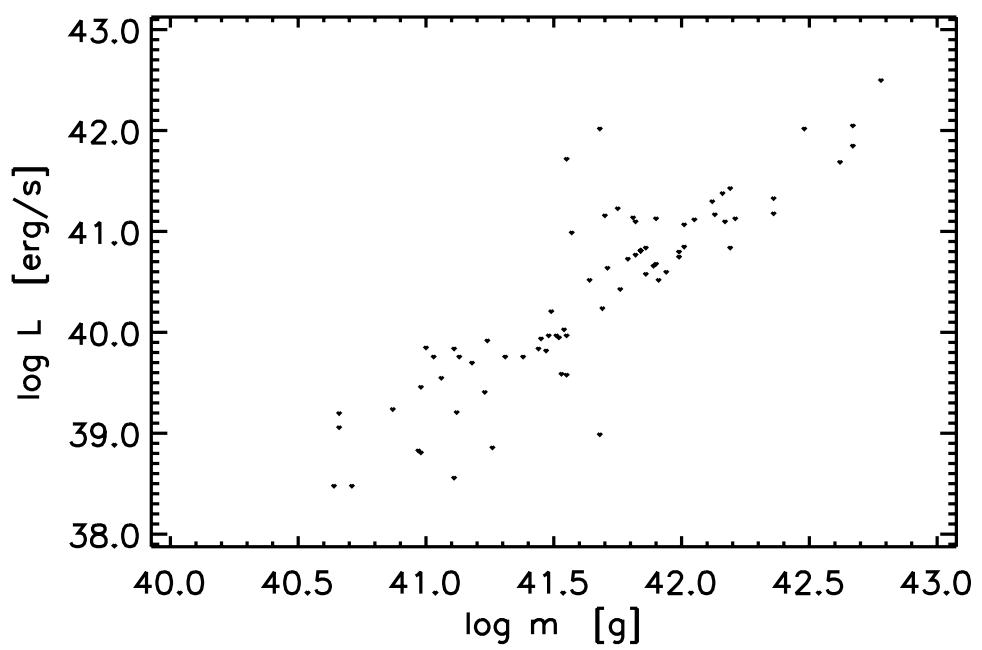

Fig. (7). "Mass - luminosity" relation for the sample of quasars.

necessary. It is interesting to note that the situation with stars is the opposite: fainter stars have smaller gravitational redshifts. This is probably due to the different slopes for stars and quasars in Fig. (6).

Finally, there is a "mass - luminosity" relation for the sample of quasars (Fig. 7, data from Table 2):

$\log \mathrm{L}_{\mathrm{q}}=-28.060+1.643 . \log \mathrm{m}_{\mathrm{q}}$, with correlation

coeff: 0.88

Comparison of quasars with stars in the Figs. (4-6) provokes a question: could there be a "bridge" between quasars and stars? This could be yet another problem for the future.

Fig. (8) shows a plot of $r_{g r} / r_{q}$ versus observed redshift. It is apparent that quantization of redshifts corresponds to a quantization of the ratio $\mathrm{r}_{\mathrm{gr}} / \mathrm{r}_{\mathrm{q}}$.

Presently, no time-dependence of the process of evolution (disintegration) could be derived. Interestingly, there is a relation "mass-density" for this sample of quasars (data from Table 2), but its cause is not yet clear:

$\log \rho_{\mathrm{q}}=77.395-1.880 . \log \mathrm{m}_{\mathrm{q}}$, with correlation coeff: -0.88

The relation shows that quasars of larger masses have smaller densities.
This relation could be explained if the speed of evolution (disintegration) depends on the mass of the quasar: quasars of larger masses evolve (disintegrate) more rapidly, thereby decreasing their density faster. Another possibility could involve extensive outer layers in massive quasars, due to excessive luminosity. If confirmed, this could provide important clues for future studies.

\section{CONCLUSIONS: UNSOLVED PROBLEMS}

Some conclusions have already been mentioned in the previous section. Here is a summary of the most important results and unsolved problems:

- A small sample of local quasars (74 quasars from 8 active galaxies) seems now to be established. All conclusions and ideas presented here concern only the sample of local quasars.

- The existence of quasars around low redshift galaxies is most likely due to ejection of the quasars from respective galaxy.

- The major part of the redshift of each quasar is probably due to gravitational reddening and larger gravitational redshifts seem to correspond to fainter objects. 


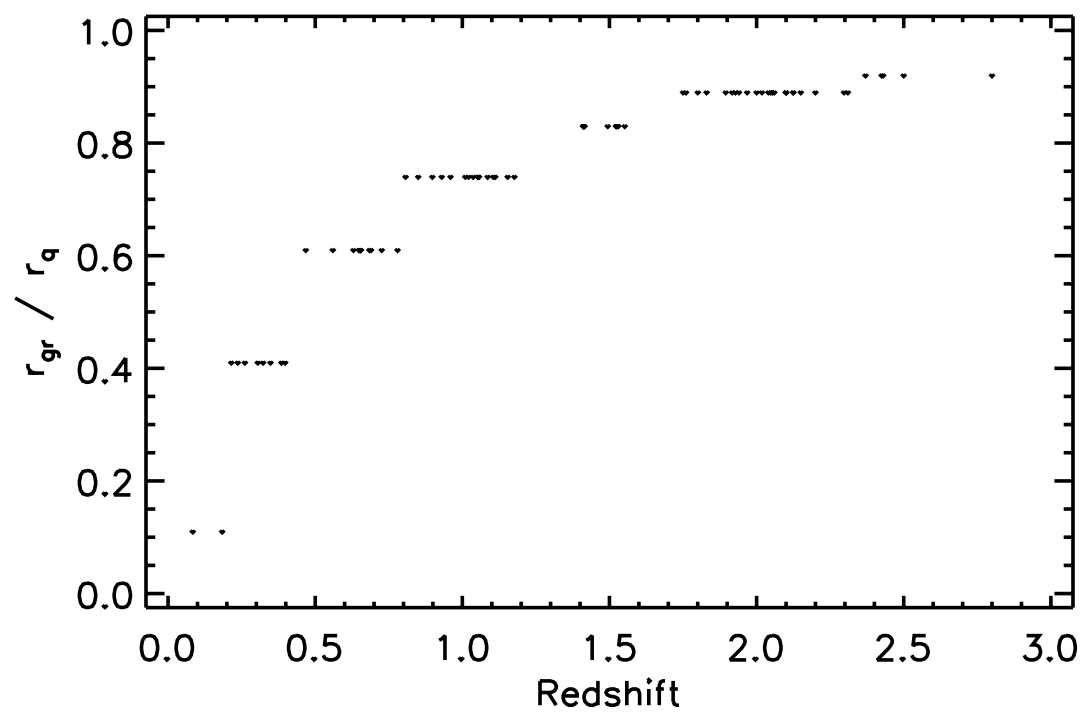

Fig. (8). Observed redshift versus $r_{g r} / r_{q}$ for the sample of quasars.

- Quasars behave like a single body, or at least the bulk of their mass is contained in a single body. That means, we deal with a new type of objects which masses could be up to $3.10^{9} \mathrm{~m}_{\circ}$ and with dimensions close to the respective gravitational radius. A theory of such strange configurations does not yet exist.

- Quasars from different galaxies are separated in radii and in luminosities. The quasars of M82 in our sample are the least luminous, and the quasars of NGC5548 are the most luminous.

- The results of this study do not contradict Arp's evolutionary scenario for quasars and Figs (2) and (3) seem to support it. However, for a confirmation of this scenario a greater sample of quasars would be necessary. If Arp's scenario is true, quasars should evolve with decreasing densities and redshifts, but with increasing radii and luminosities. At large redshifts, the evolution of redshift to lower values as density decreases is very fast. This may explain the well known decline of number of quasars with $\mathrm{z}>3$. The end-product of quasar-evolution would be smallmass galaxies.

- The evolution of quasars into galaxies may explain the well known fact of galaxies, hosting a quasar. It may be just the opposite: the quasar hosts the surrounding galaxy, because the quasar was the origin.

- The above scenario poses another fundamental question. If the end-product of quasar evolution could be galaxies, we could expect that also galaxies, which are in transition (i.e. their densities and redshifts are still decreasing), to have some small component in their redshifts, due to gravitational reddening. Then it appears that we should take care to study the Hubble diagram with respect to possible gravitational redshifts. Whether or not gravitational redshifts could change the value of the Hubble constant remains to be seen. Considerations about possible evolution of the Hubble diagram have been given by Arp [44].

- Relationships are presented for: "density-redshift", "absolute mag -radius", "absolute mag - mass", "mass - luminosity", and "mass - radius" for the sample of quasars. Their interpretation, however, is not yet clear.

- A "mass - density" relation is found, which could be explained in terms of faster evolution (disintegration) of quasars of larger masses, or else in terms of extensive outer layers in massive quasars.

- An important, yet unsolved problem is the Karlsson's sequence, possibly involved with the gravitational redshifts. The quantization of gravitational redshifts is a new phenomenon with possible implications for other physical properties of quasars (see Fig. 8). Another implication: the reduction of redshifts of quasars to the redshift of the parent galaxy was considered here as reduction for the cosmological redshift. This could be exact only if we can first reduce the gravitational redshift and the Doppler redshift of the galaxy itself.

- A related problem is the way evolution of quasars proceeds: continuous, or step-by-step? In 1997, Halton Arp [28] suggested that redshifts drop in steps, each step corresponding to the next lower value of the Karlsson's sequence. From Fig (8), this could not be ruled out, but the implications for other physical characteristics of quasars are yet to be studied.

Ejection of quasars, their physical properties, and their evolution present a serious challenge to present day astrophysics. Ignoring problems never helps to solve them. It is to be expected that in the near future new studies will help to increase the sample of local quasars. At some point, if the above findings are confirmed with large enough number of local quasars, the introduction of radically new ideas will become unavoidable.

\section{ACKNOWLEDGEMENT}

For this work I used the catalogue of Veron-Cetty and Veron, 13 th ed. by the CDS - Strassbourg.

\section{REFERENCES}

[1] Antonucci R. Unified models for active galactic nuclei and quasars. AR Astron Astrophys 1993; 31: 473-521. 
[2] Djorgowski SG, Volonteri M, Springel V, Bromm V, Meylan G. The origins and the early evolution of quasars and supermassive black holes. Proceedings of XI Marcel Grossmann Meeting on General Relativity 2008.

[3] Kembhavi AK, Narlikar JV. Quasars and Active Galactic Nuclei: an Introduction. Cambridge: Cambridge University Press, 1999.

[4] Lopez-Corredoira M. Pending problems in QSOs. 2009.

[5] Lopez-Corredoira M. Observational Cosmology: caveats and open questions in the standard model. In Recent Research Developments in Astron and Astrophys, arXiv:astro-ph/0310214.

[6] Hoyle F, Fowler WA. On the nature of strong radiosources. Mon Not R Astron Soc 1963; 125: 169-176.

[7] Yamada SF, Shioya Y, Taniguchi Y, et al. Are Two z $\sim 6$ Quasars Gravitationally Lensed? Publ Astron Soc Japan 2003; 55: 733-8.

[8] Richards GT, Strauss MA, Pindor B, et al. A Snapshot Survey for Gravitational Lenses among $\mathrm{z}>4.0$ Quasars. I. The $\mathrm{z}>5.7$ Sample. Astron J 2004; 127: 1305-12.

[9] Wilson OC. Possible applications of supernovae to the study of the nebular red shifts. Astrophys J 1939; 90: 634-6.

[10] Hawkins MRS. Time Dilation and Quasar Variability. Astrophys J 2001; 553: L97-L100.

[11] Karlsson KG. Possible discretization of quasar redshifts. Astron Astrophys 1971; 13: 333-5.

[12] Karlsson KG. On the existence of significant peaks in the quasar redshift distribution. Astron Astrophys 1977; 58: 237-40.

[13] Burbidge G, Napier WM. The distribution of redshifts in new samples of quasi-stellar objects. Astron J 2001; 121: 21-30.

[14] Arp H, Bi H, Chu Y, Zhu X. Periodicity of quasar redshifts. Astron Astrophys 1990; 239: 33-49.

[15] Arp HC. QSOs, redshifts and controversies. Berkeley, USA: Interstellar Media 1987.

[16] Arp HC. Catalogue of discordant redshift associations. Montreal, Canada: Apeiron 2003.

[17] Burbidge GR. Noncosmological redshifts. Publ Astron Soc Pacific 2001; 113: 899-902.

[18] Bell MB. Further evidence for large intrinsic redfshifts. Astrophys J 2002; 566: 705-11.

[19] Lopez-Corredoira M, Gutierrez CM. Research on candidates for non-cosmological redshifts. AIP Conf Proc of First Crisis in Cosmology Conf. Lerner EJ, Almeida JB, Eds. NY, USA: AIP 2006; 822: 75-92.

[20] Lopez-Corredoira M, Gutierrez CM. The field surrounding NGC7603: Cosmological or non-cosmological redshifts? Astron Astrophys 2004; 421: 407-22.

[21] Arp H. Identification of X-ray sources $<1^{\circ}$ from Seyfert galaxies. Astron Astrophys 1997; 319: 33-44.

[22] Arp H. Pairs of X-ray sources across Seyferts: the NGC4235 field. Astron Astrophys 1997; 328: L17-L20.
[23] Arp H. A QSO 2.4 arcsec from a dwarf galaxy-the rest of the story. Astron Astrophys 1999; 341: L5-L8.

[24] Lopez-Corredoira M, Gutierrez CM. First tentative detection of anisotropy in the QSO distribution around nearby edge-on spiral galaxies. Astron Astrophys 2007; 461: 59-69.

[25] Burbidge EM. Spectra of two quasars possibly ejected from NGC4258. Astron Astrophys 1995; 298: L1-L4

[26] Burbidge EM. Spectra of Two X-Ray emitting Quasi-stellar Objects Apparently Ejected from the Seyfert Galaxy NGC2639. Astrophys J 1997; 484: L99-L101.

[27] Burbidge EM, Burbidge G. Ejection of Matter and Energy from NGC4258. Astrophys J 1997; 477: L13-L15.

[28] Arp H. Quasar Creation and Evolution into Galaxies. J Astrophys Astron 1997; 18: 393-406.

[29] Fan X, Narayanan VK, Lupton RH, et al. A Survey of Z > 5.8 Quasars in the Sloan Digital Sky Survey I. Discovery of three new quasars and the spatial density of luminous quasars at $\mathrm{z} \sim 6$. Astron J 2001; 122: 2833-49.

[30] Simon LE, Hamann FW, Pettini M. Physical properties of absorbers in high redshift quasars. Rev Mex Astron C 2007; 29: 177.

[31] Greenstein JL, Schmidt M. The quasi-stellar radio sources 3C48 and 3C273. Astrophys J 1964; 140: 1-37.

[32] Narlikar JV. Two astrophysical applications of conformal gravity. Ann Phys 1977; 107: 325-336.

[33] Narlikar JV, Arp HC. Flat Spacetime Cosmology- a Unified Framework for Extragalactic Redshifts. Astrophys J 1993; 405: 516.

[34] Veron-Cetty M-P, Veron P. A catalogue of quasars and active nuclei: $13^{\text {th }}$ edition. Astron Astrophys 2010; 518A: A10.

[35] Burbidge EM. A group of quasi-stellar objects closely associated with NGC1068. Astrophys J 1999; 511: L9-L11.

[36] Burbidge EM, Burbidge G, Arp HC, Zibetti S. QSOs associated with M82. Astrophys J 2003; 591: 690-694.

[37] Burbidge EM, Burbidge G, Arp HC, Napier WM. An anomalous concentration of QSOs around NGC3079. 2005; astro-ph10815 .

[38] Arp H, Burbidge EM, Chu Y, et al. NGC3628: ejection activity associated with quasars. Astron Astrophys 2002; 391: 833-840.

[39] Burbidge EM, Burbidge G. QSOs in the field of the Seyfert1 galaxy NGC5548. Publ Astron Soc Pacific 2002; 114: 253-56.

[40] Arp H. Ultraviolet excess objects in the region of a companion galaxy to NGC2639. Astrophys J 1980; 236: 63-9.

[41] Burbidge G. Two universes. Astrophys Sp Sci 1996; 244: 169-76.

[42] Arp Halton. Evolution of quasars into galaxies and its implications for the birth and evolution of matter. APEIRON 1998; 5: 135-142.

[43] Arp H. Association of x-ray quasars with active galaxies. IAUS 183 1999; 290-5.

[44] Arp H. Quasars and the Hubble Relation. 2007; arXiv: 0711.2607: $1-10$

Received: September 15, 2010

Revised: November 10, 2010

Accepted: November 15, 2010

(C) K. P. Panov; Licensee Bentham Open.

This is an open access article licensed under the terms of the Creative Commons Attribution Non-Commercial License (http://creativecommons.org/licenses/by-nc/3.0/) which permits unrestricted, non-commercial use, distribution and reproduction in any medium, provided the work is properly cited. 